\title{
Tiny human stomachs grown in the lab
}

Artificial guts could be used to study diseases and test drug treatments.

\section{Mark Zastrow}

29 October 2014

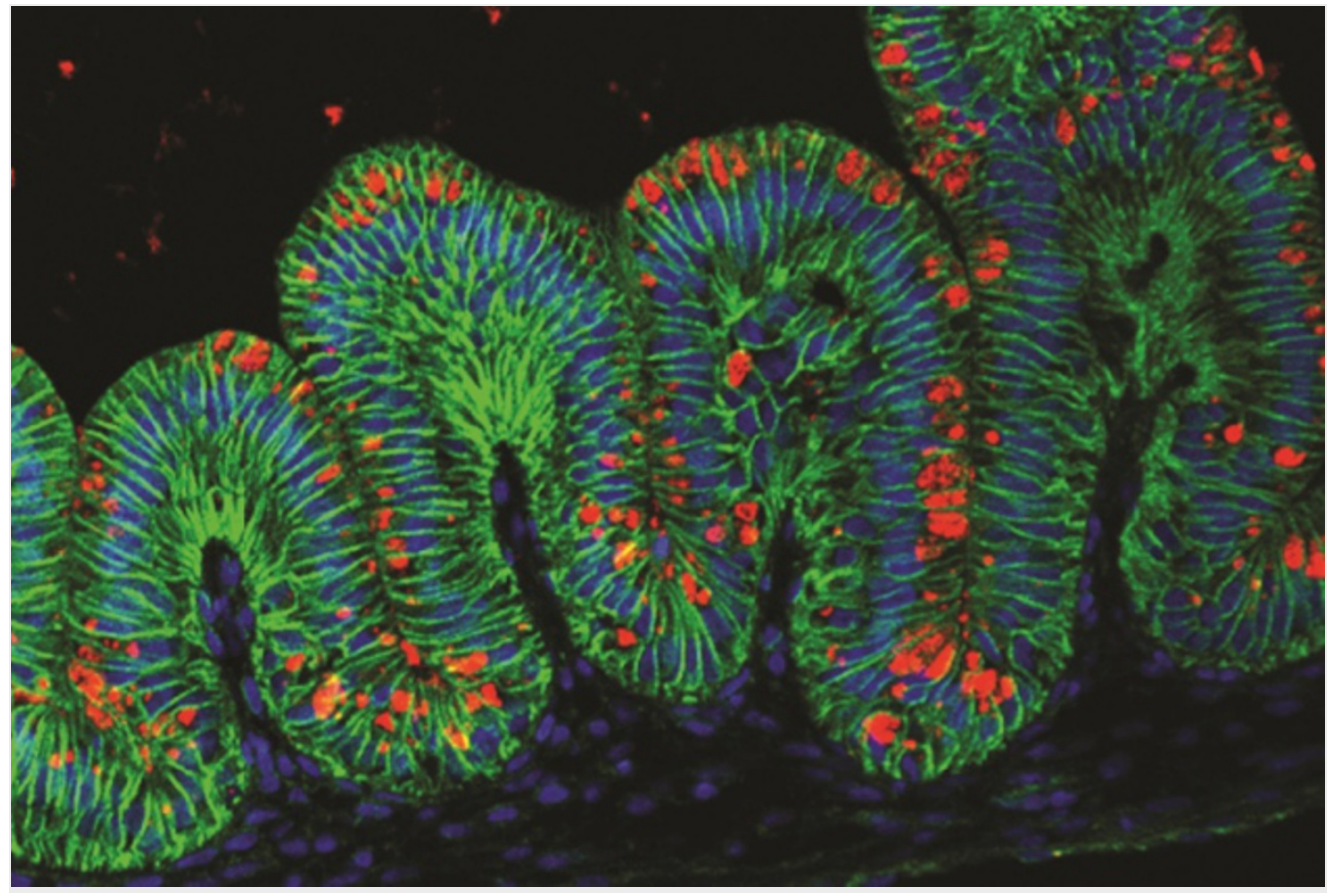

Kyle McCracken

Part of a miniature stomach grown in the lab, stained to reveal various cells found in normal human stomachs.

Scientists have successfully grown miniature stomachs in the lab from human stem cells, guiding them through the stages of development seen in an embryo. The lumps of living tissue, which are no bigger than a sesame seed, have a gland structure that is similar to human stomachs and can even harbour gut bacteria.

The feat, reported in this week's Nature ${ }^{1}$, offers a window to how cells in human embryos morph into organs. Scientists say that these 'gastric organoids' could also be used to understand diseases such as cancer, and to test the stomach's response to drugs.

"This is extremely exciting," says Calvin Kuo, a stem-cell biologist at Stanford University in California. "To be able to recapitulate that in a dish is quite a technical achievement." 
The stem cells used to grow the mini stomachs are pluripotent, or plastic: given the right environment, they can mature into any type of cell. But to coax them down a specific path in the lab requires recreating the precise sequence and timing of environmental cues in the womb the signals from proteins and hormones that tell cells what kind of tissue to become. Bits of kidney, liver, brain and intestine have previously been grown in a lab dish using this technique.

\section{Stomach switch}

The key to turning pluripotent stem cells into stomach cells was a pathway of interactions that acts as a switch between growing tissues in the intestine and in the antrum, a part of the stomach near its outlet to the small intestine.

When the stem cells were around three days old, researchers added a cocktail of proteins including Noggin, which suppresses that pathway, and timed doses of retinoic acid, a compound in vitamin A. After nine days, the cells were left to grow in a protein bath.

At 34 days, the resulting organoids were only a few millimetres in diameter and had no blood cells, immune cells, nor the ability to process food or secrete bile. But their gland structures and each marker of their development paralleled development in their control tissues, which the team obtained from mice. In that sense, they "are remarkably similar to an actual stomach", says study leader James Wells, a developmental biologist at Cincinnati Children's Hospital Medical Center in Ohio.

That similarity allowed the researchers to use the tiny stomachs as test subjects for human disease by injecting them with Helicobacter pylori, a bacterium that targets the antrum and can cause ulcers and stomach cancer. Within 24 hours, the team found that $H$. pylori was causing the organoid cells to divide twice as fast as normal, and activating a particular gene, c-Met, that can cause tumours. These effects are also seen in human stomachs infected with $H$. pylori.

The researchers say that they can grow the stomach organoids from both embryonic stem cells and skin cells induced to pluripotency. Jason Mills, a gastrointestinal pathologist at Washington University School of Medicine in St. Louis, envisions growing thousands of such organoids, each from a different person's cells, and infecting them with a pathogen to study the role of individual genetics.

Wells says that his team's long-term goal is to be able to grow personal stomach tissue to patch up ulcers in humans. He and some colleagues are already attempting to use human organoids to plug stomach holes in mice.

Nature I doi:10.1038/nature.2014.16229

\section{References}

1. McCracken, K. W. et al. Nature http://dx.doi.org/10.1038/nature13863 (2014). 